Hautarzt 2022 $\cdot 73: 556-559$

https://doi.org/10.1007/s00105-021-04898-y

Angenommen: 15. September 2021

Online publiziert: 5 . Oktober 2021

(c) Der/die Autor(en) 2021

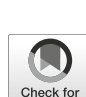

Check for
updates

\section{Haarwachstum unter Dupilumab bei Alopecia areata universalis und atopischer Dermatitis}

\author{
L. Reinhold $\cdot$ K. Lange $\cdot$ H. Lindhof $\cdot$ B. Homey $\cdot$ P. Firouzi-Memarpuri \\ Heinrich-Heine-Universität, Medizinische Fakultät, Klinik für Dermatologie, Universitätsklinikum \\ Düsseldorf, Düsseldorf, Deutschland
}

\title{
Zusammenfassung
}

Berichtet wird über eine 46-jährige Patientin, die seit ihrer frühen Kindheit unter einer schweren atopischen Dermatitis leidet und seit ihrem 18. Lebensjahr unter einer Alopecia areata totalis, die mittlerweile in eine Alopecia areata universalis übergegangen ist. Durch die Einleitung einer Therapie mit dem monoklonalen Antikörper Dupilumab wurde erneutes Haarwachstum am Kapillitium, im Gesicht und an den Unterschenkeln beobachtet. Dupilumab blockiert die a-Untereinheit des IL(Interleukin)-4-Rezeptors, unterbindet die Signalkaskade von IL-4 und IL-13 und führt so zu einer reduzierten Th2-Immunantwort. Die schwere Ausprägung der Ekzeme und der Juckreiz mit Ein- und Durchschlafstörungen sind bereits 14 Tage nach Beginn der Einnahme zurückgegangen. Die Patientin verträgt das Medikament ohne wesentliche Nebenwirkungen, ihre Lebensqualität ist deutlich verbessert. Patienten mit einer schweren atopischen Dermatitis und einer Alopecia areata könnten von Dupilumab in Zukunft doppelt profitieren.

\section{Schlüsselwörter}

Alopecia areata totalis · Alopecia areata universalis · Dupilumab · Monoklonaler Antikörper · Atopische Dermatitis

\section{Anamnese}

Eine 46-jährige Patientin stellte sich mit seit dem 18. Lebensjahr rezidivierenden Episoden einer Alopecia areata totalis vor, die vor einem halben Jahr in eine Alopecia areata universalis übergegangen sei.

Multiple Therapien mit Glukokortikosteroid-haltigen Externa seien erfolglos geblieben. Bis dato seien keine weiteren topischen Behandlungen, wie beispielsweise mit Diphenylcyclopropenon, oder Systemtherapien mit Prednisolon/ Dexamethason, eingeleitet worden.

Begleitend bestehe eine schwere atopische Dermatitis am gesamten Integument mit starkem, den Schlaf deutlich einschränkenden Juckreiz. Diese sei in rezidivierenden Schüben seit der frühen Kindheit bekannt.

Weitere Begleiterkrankungen seien eine Hypothyreose sowie eine chronische Sinusitis.

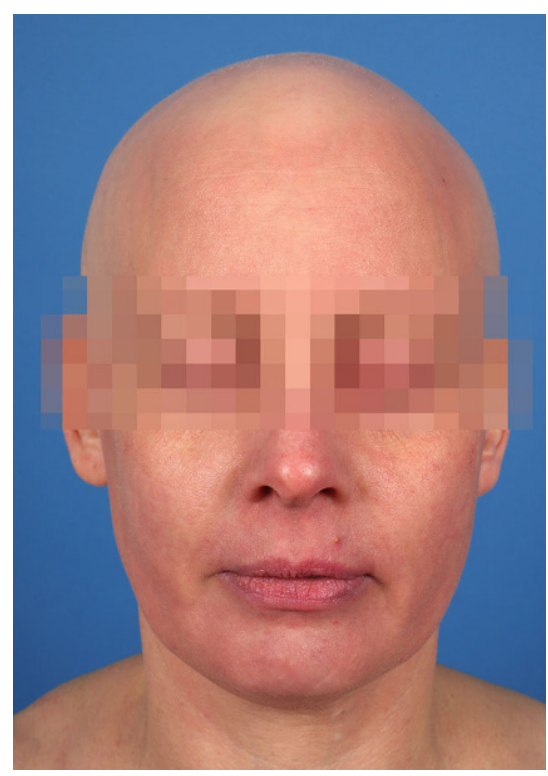

Abb. $1 \triangle$ Alopecia areata totalis 

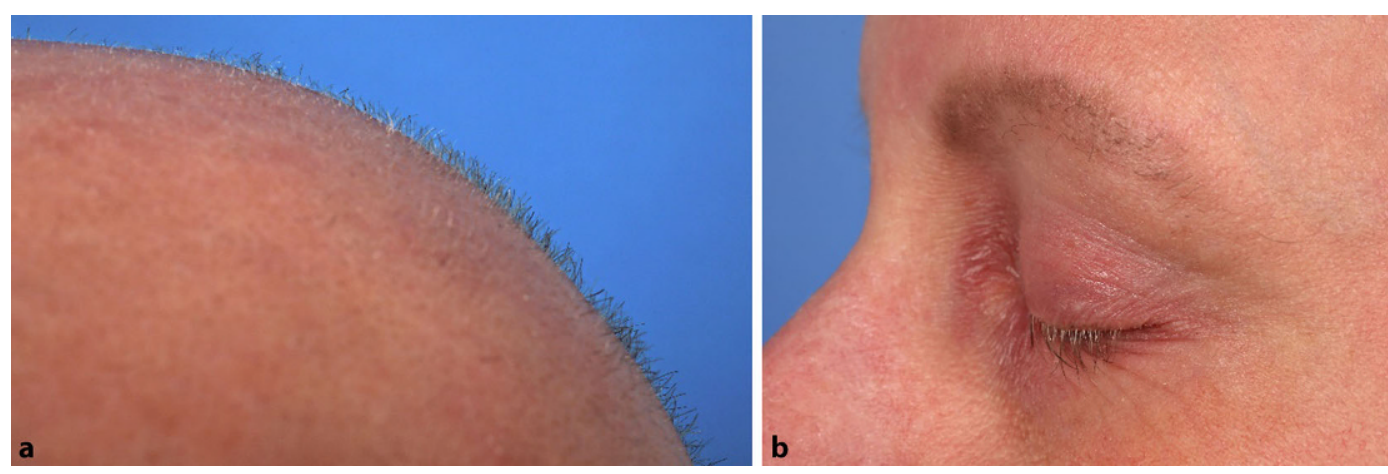

Abb. $2 \varangle$ a Tangentialaufnahme vom Kapillitium. Multiple pigmentierte und depigmentierte Haare. b Pigmentierte Haare im Bereich von Augenbrauen und Wimpern

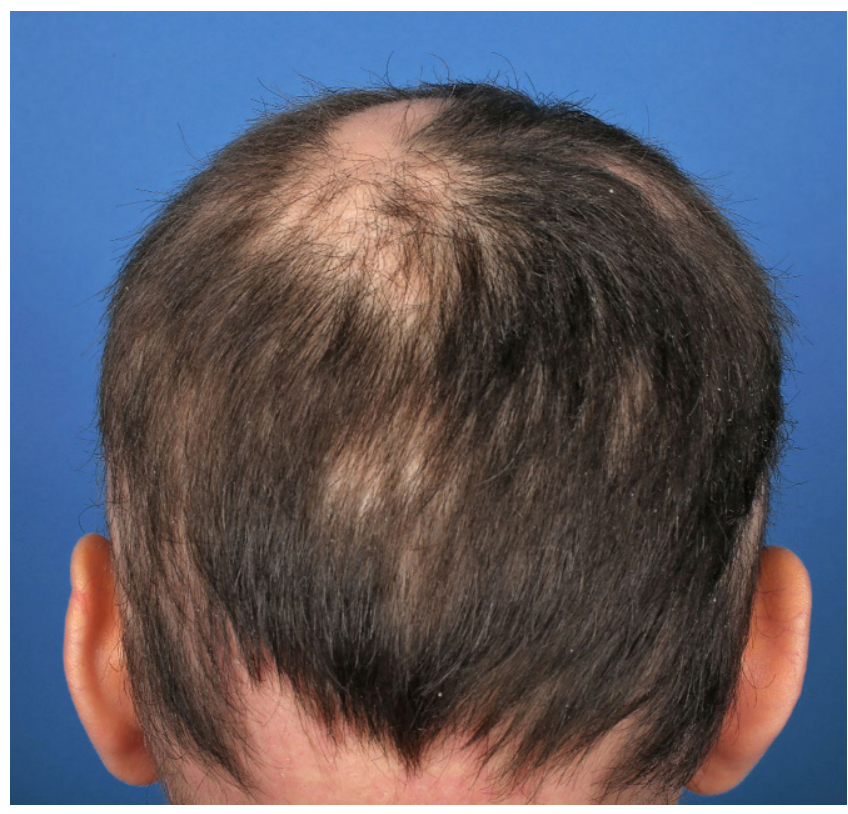

Abb. $3 \triangleleft$ Flächiges Haarwachstum 16 Wochen nach Einleitung von Dupilumab

Dauermedikation: Substitution von L-Thyroxin, Selen, Zink und Vitamin D3, Einnahme von Antihistaminika.

\section{Befund}

Bei Erstvorstellung zeigte sich das gesamte Integument inklusive der Partie der Augenbrauen, der Wimpern und des Intimbereichs haarlos (-Abb. 1). Des Weiteren fanden sich am kompletten Integument mit Betonung der großen Gelenkbeugen multiple erythematöse, teilweise stark kratzexkoriierte Papeln und Plaques. Außerdem zu sehen waren eine starke Lichenifikation und positive Atopiestigmata, wie z. B. eine Dennie-Morgan-Falte und eine ausgeprägte Xerosis cutis. Im Bereich der Fingernägel präsentierten sich multiple Tüpfel. Insgesamt betrug der SCORAD 60, der DLQI 24.

\section{Diagnostik}

Auflichtmikroskopisch zeigte sich eine reizlose Kopfhaut mit vorhandenen Follikelöffnungen. Da es sich sowohl bei der Alopecia areata als auch bei der atopischen Dermatitis im weitesten Sinne um eine Blickdiagnose handelt, wurde in diesem Fall auf eine Probebiopsie zur histopathologischen Untersuchung verzichtet.

Laborchemisch zeigten sich bei den bestimmten Routineparametern (Elektrolyte, Nieren-, Leber- und Schilddrüsenwerte, kleines Blutbild, Differenzialblutbild) keine Auffälligkeiten.

\section{Therapie und Verlauf}

Aufgrund der schweren atopischen Dermatitis leiteten wir umgehend eine Therapie mit dem monoklonalen Antikörper Dupilumab ein. Er blockiert die a-Untereinheit des IL(Interleukin)-4-Rezeptors und unter- bindet die Signalkaskade von IL-4 und IL13. Dies führt zu einer reduzierten Th2Immunantwort [1].

Als topische Begleittherapie erhielt die Patientin Mometasonfuroat-haltige Externa für die betroffenen ekzematösen Körperstellen. Initial wurde Dupilumab in einer Dosierung von $600 \mathrm{mg}$ s.c. verabreicht, im Anschluss $300 \mathrm{mg}$ s.c. alle 14 Tage.

Bereits 6 Wochen nach der ersten Dupilumab-Gabe zeigte sich ein Haarwachstum im Bereich des Kapillitiums und des Gesichts (-Abb. 2a,b). Die Haare waren teils depigmentiert, teils bräunlich pigmentiert. Wenig später präsentierten sich feine, dunkel pigmentierte Haare an beiden Unterschenkeln. Weitere 10 Wochen später zeigte sich ein deutlicher, flächiger Haarwuchs mit nur noch wenigen haarlosen Arealen (- Abb. 3). Zu dieser Zeit lag der SCORAD bei 8,5 und der DLQI bei 5 .

Bis auf eine leichte Konjunktivitis, die sich mit Tränenersatzmittel erfolgreich behandeln ließ, hatte die Patientin keine Nebenwirkungen und verträgt das Medikament bis dato hervorragend.

Bezüglich der atopischen Dermatitis ist anzumerken, dass die Patientin bereits 14 Tage nach Einleitung von Dupilumab einen deutlichen Rückgang des Juckreizes und wenig später auch des Ekzems verspürte und keine Ein- bzw. Durchschlafstörungen mehr aufwies.

Aktuell pflegt die Patientin intensiv mit rückfettenden Externa mehrmals täglich und kann auf topische Glukokortikosteroide verzichten.

\section{Kommentar}

Unter einer Alopecia areata versteht man einen akut einsetzenden, entzündlich bedingten, symptomlosen, meistens kreis- 
rund beginnenden Haarausfall, bei dem die Haarfollikel intakt bleiben ohne zu vernarben. Männer und Frauen sind in etwa gleich häufig betroffen. Ein Häufigkeitsgipfel wird in der zweiten und dritten Lebensdekade beobachtet [2]. Die häufigste Lokalisation ist das Kapillitium. Hier reicht die Vielfalt der Erkrankung von einem oder mehreren solitären haarlosen Arealen (Alopecia areata localisata) über den kompletten Verlust der Kopfbehaarung (Alopecia areata totalis) bis hin zum kompletten Verlust der Körperbehaarung (Alopecia areata universalis). Die Alopecia areata ist auch ohne therapeutische Interventionen bei leichter Ausprägung meist reversibel.

Eine kausale Therapieoption gibt es bis dato nicht. Therapieversuche können von topischen bzw. intraläsionalen Glukokortikosteroiden, topischem Diphenylcyclopropenon (DCP) oder Dithranol über systemische Glukokortikosteroide bis hin zu Glukokortikosteroid-Stoßtherapien reichen. Ausgeprägte Befunde zeigen sich dabei häufig therapierefraktär.

Die Alopecia areata weist eine Lebenszeitinzidenz von etwa 2,1\% auf [3].

Die Ätiologie ist bis heute noch nicht vollständig verstanden. Neben möglichen autoimmunologischen Ursachen, infektallergischen oder auch psychischen Faktoren wird ein vermehrtes Auftreten bei atopischer Diathese beobachtet [2]. Durch den Verlust des Immunprivilegs kommt es zu einem autoimmunvermittelten Angriff des Haarfollikels [2]. Da auch hierbei die IL-4- und IL-13-vermittelteTh2-Immunantwort eine Rolle spielt, liegt der immunologische Bezug zur atopischen Dermatitis und gleichzeitig zur Wirkung des Antikörpers Dupilumab nahe.

In der Literatur gibt es mehrere Einzelfallberichte dazu, dass es, wie bei der hier vorgestellten Patientin, unter der Therapie mit Dupilumab zu einem Haarwachstum bei Alopecia areata gekommen ist $[4,5]$.

Aufgrund des bei der Alopecia areata möglichen spontan wiedereinsetzenden Haarwuchs besteht grundsätzlich die Möglichkeit eines zufälligen zeitlichen Zusammenhangs bezüglich des Haarwachstums und der Einnahme von Dupilumab.

Ein weiterer spekulativer Diskussionspunkt wäre das Auslösen der Alopecia areata durch die schwere atopische Dermatitis als spezifischer Auslöser bzw. Stressor.

\section{Hair growth with dupilumab in alopecia areata universalis and atopic} dermatitis

We report the case of a 46-year-old woman who has suffered from severe atopic dermatitis since early childhood and from alopecia areata totalis since she was 18 years old, which has now developed into alopecia areata universalis. After the introduction of therapy with the monoclonal antibody dupilumab, renewed hair growth of the scalp, face and lower legs was observed. Dupilumab blocks the a-subunit of interleukin (IL)-4 receptor and prevents the signaling cascade of IL-4 and IL-13. This leads to a reduction of Th2 immune response. The severe eczema and itching with difficulties falling and staying asleep decreased after just 14 days. The patient tolerates the drug without significant side effects and has a significantly improved quality of life. Patients with severe atopic dermatitis and alopecia areata could benefit twice from the use of dupilumab in the future.

\section{Keywords}

Alopecia areata totalis · Alopecia areata universalis · Dupilumab · Monoclonal antibody · Atopic dermatitis

Durch die erfolgreiche Behandlung der ursächlichen atopischen Dermatitis komme es demnach zu einem Ausschalten des Stressors und wiederum zu einem Neuwachsen der Haare. In diesem Fall wäre der Wirkstoff Dupilumab nur indirekt für die Remission der Alopecia areata verantwortlich.

Der ebenfalls für die atopische Dermatitis zugelassene JAK-Inhibitor Baricitinib erzielte kürzlich in einer Phase-3-Studie bei Patienten mit schwerer Alopecia areata eine statistisch signifikante Verbesserung des Haarwachstums auf der Kopfhaut im Vergleich zu den Patienten, die mit Placebo behandelt wurden [6].

\section{Fazit für die Praxis}

- Der monoklonale Antikörper Dupilumab könnte eine neue Therapieoption für $\mathrm{Pa}$ tienten mit einer Alopecia areata darstellen.

- Patienten, die parallel eine schwere atopische Dermatitis aufweisen, könnten sogar doppelt profitieren.
Korrespondenzadresse

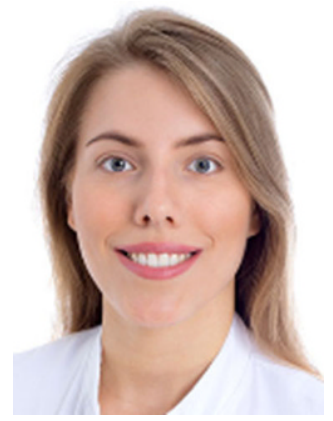

Dr. med. L. Reinhold

Heinrich-Heine-Universität, Medizinische Fakultät, Klinik für Dermatologie, Universitätsklinikum Düsseldorf Moorenstr. 5, 40225 Düsseldorf, Deutschland Lara.Reinhold@med.uni-duesseldorf.de

Funding. Open Access funding enabled and organized by Projekt DEAL.

\section{Einhaltung ethischer Richtlinien}

Interessenkonflikt. L. Reinhold, K. Lange, H. Lindhof, B. Homey und P. Firouzi-Memarpuri geben an, dass kein Interessenkonflikt besteht.

Für diesen Beitrag wurden von den Autoren keine Studien an Menschen oder Tieren durchgeführt. Für die aufgeführten Studien gelten die jeweils dort angegebenen ethischen Richtlinien. Für Bildmaterial oder anderweitige Angaben innerhalb des Manuskripts, über die Patienten zu identifizieren sind, liegt von innen und/oder ihren gesetzlichen Vertretern eine schriftliche Einwilligung vor.

Open Access. Dieser Artikel wird unter der Creative Commons Namensnennung 4.0 International Lizenz veröffentlicht, welche die Nutzung, Vervielfältigung, Bearbeitung, Verbreitung und Wiedergabe in jegli- 
chem Medium und Format erlaubt, sofern Sie den/die ursprünglichen Autor(en) und die Quelle ordnungsgemäß nennen, einen Link zur Creative Commons Lizenz beifügen und angeben, ob Änderungen vorgenommen wurden.

Die in diesem Artikel enthaltenen Bilder und sonstiges Drittmaterial unterliegen ebenfalls der genannten Creative Commons Lizenz, sofern sich aus der Abbildungslegende nichts anderes ergibt. Sofern das betreffende Material nicht unter der genannten Creative Commons Lizenz steht und die betreffende Handlung nicht nach gesetzlichen Vorschriften erlaubt ist, ist für die oben aufgeführten Weiterverwendungen des Materials die Einwilligung des jeweiligen Rechteinhabers einzuholen.

Weitere Details zur Lizenz entnehmen Sie bitte der Lizenzinformation auf http://creativecommons.org/ licenses/by/4.0/deed.de.

\section{Literatur}

1. Simpson EL, Bieber T, Guttman-YasskyE et al (2016) Two phase 3 trials of dupilumab versus placebo in atopic dermatitis. NEngl J Med 375:2335-2348

2. Strazzulla LC et al (2018) Alopecia areata: disease characteristics, clinical evaluation, and new perspectives on pathogenesis. J Am Acad Dermatol 78(1):1-12

3. Mirzoyev SA et al (2014) Lifetime incidence risk of alopecia areata estimated at 2.1 percent by Rochester Epidemiology Project, 1990-2009. JInvest Dermatol 134(4):1141-1142

4. Penzi LR et al (2018) Hair regrowth in a patient with long-standing alopecia totalis and atopic dermatitis treated with dupilumab. JAMA Dermatol 154(11):1358-1360

5. Alniemi DT, McGevna L (2019) Dupilumab treatment for atopic dermatitis leading to unexpected treatment for alopecia universalis. JAAD Case Rep 5:111-112

6. Eli Lilly and Company (2018) A study of baricitinib (LY3009104) in participants with severe or very severe alopecia areata (BRAVE-AA1). NCT number: NCT03570749

\section{Möchten Sie einen Beitrag für die Zeitschrift Die Dermatolo- gie einreichen?}

Wir freuen uns, dass Sie unsere Zeitschrift Die Dermatologie mitgestalten möchten. Um Ihnen bei der Manuskripterstellung behilflich zu sein, haben wir für unsere Autorinnen und Autoren Hinweise zusammen gestellt, die Sie im Internet finden unter www.springer.com/journal/105 (For Authors).

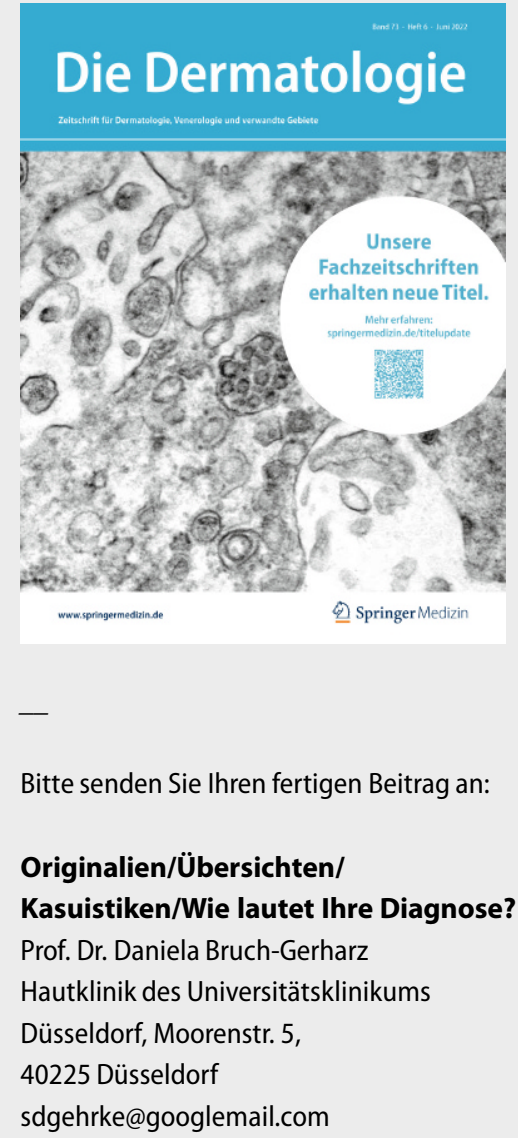

Dermatoskopie - Fall des Monats:

Prof. Dr. Andreas Blum

Hautarztpraxis Konstanz und Lehrpraxis der

Medizinischen Fakultät Universität Tübingen

Augustinerplatz 7

78462 Konstanz

a.blum@derma.de

\section{One Minute Wonder:}

PD Dr. Dr. Alexander Zink, Dr. Sarah Preis

Klinik und Poliklinik für Dermatologie und Allergologie am Biederstein,

TU München

Bitte schreiben Sie bei Fragen an

die Verlagsredaktion:

julie.kind@springer.com

\section{CME Zertifizierte Fortbildung:}

Anfragen an:

Prof. Dr. Evelyn Gaffal

Universitätshautklinik

Universitätsklinikum Magdeburg

Labor für Experimentelle Dermatologie

Leipziger Straße 44

39120 Magdeburg

evelyn.gaffal@med.ovgu.de

Prof. Dr. Sonja Ständer

Klinik und Poliklinik für Hautkrankheiten,

Universitätsklinikum Münster

Von-Esmarch-Str. 58

48149 Münster

sonja.staender@uni-muenster.de

Prof. Dr. Rolf-Markus Szeimies

Klinik für Dermatologie und Allergologie, Knappschaftskrankenhaus Recklinghausen, Klinikum Vest $\mathrm{GmbH}$,

Dorstener Str. 151,

45657 Recklinghausen

dermatologie@kk-recklinghausen.de

PD Dr. Dr. Alexander Zink

Klinik und Poliklinik für Dermatologie und

Allergologie am Biederstein,

Technische Universität München

Biedersteiner Str. 29

80802 München

alexander.zink@tum.de 